


\title{
LOCALLY COMPACT TOPOLOGIES ON A GROUP AND THE CORRESPONDING CONTINUOUS IRREDUCIBLE REPRESENTATIONS
}

\author{
KLAUS BichteleR
}

It is shown that two different topologies on a group $G$ both of which make it into a locally compact group, usually give rise to different continuous irreducible unitary representations. To be more precise: If the continuous irreducible unitary representations of $G$ coincide for the two topologies, then these topologies are the same in the following cases: The topologies are comparable; There exists a normal subgroup of $G$, open and $\sigma$-compact in one of the topologies.

In the case of an abelian locally compact group $G$, complete information about its structure is contained in its dual object $\hat{G}$, which is, again, an abelian locally compact group. If $G$ is an arbitrary locally compact group, its dual $\widehat{G}$ is a topological space only-the space of equivalence classes of continuous irreducible unitary representations of $G$, furnished with an intricate topology. There is the natural question of how much information about $G$ is contained in this space $\hat{G}$. This paper is an attempt to answer a very restricted problem in this context, namely the following: does the 'functor' $\wedge$ distinguish between two different topologies $\rho$ and $\sigma$ on an abstract group, each of which makes it into a locally compact group? To be more precise, we shall show (see $\S 9$ below) that in a large class of cases the following is true: If the irreducible representations of $G$ continuous with respect to $\rho$ coincide with those continuous with respect to $\sigma$, then $\rho=\sigma$. A special result of this sort has been obtained in [2], and we list it here for reference as

Proposition 1. If every irreducible representation of the locally compact group $G$ is continuous, then $G$ is discrete.

The paper is divided into eight more sections, of which the first contain some simple lemmas about locally compact groups, mainly listed for reference, while the later ones treat special cases of pairs of locally compact topologies on a group with the same continuous irreducible representations. A survey of the results obtained is given in $\$$.

I owe to Professor K. Ross the remark that slightly stronger, analogous results, due to I. Kaplansky are known for some time in the Abelian case $^{1)}$.

1) (cf. [6, Corollary 24]; [10], [15]). 
2. Generalities on locally compact topologies on a group. The simple lemmas of this section will permit us below to reduce questions of weak equivalence or equality of topologies on a group to the same questions on subgroups and quotient groups. For the following three subsections, $G$ denotes a locally compact group.

2.1. The identity component $G_{0}$ of $G$ is the intersection of all open subgroups of $G$, and $G$ is totally disconnected, if and only if there exists a basis at $e$ of open compact subgroups $([10, \S 7]$ or [14, p. $54 \mathrm{ff}]$.$) . From this it follows immediately that any subgroup of$ $G$ and any quotient of $G$ by a closed normal subgroup is totally disconnected, if $G$ is. We recall that if $G$ is compact and totally disconnected, the open and normal subgroups form a basis at $e$.

Lemмa 2.2. The following statements are equivalent:

(i) $G$ is o-compact.

(ii) $G$ is a Lindelöf space.

(iii) If $V$ is a neighbourhood of the identity, $G$ can be covered by countably many left (right) translates of $V$.

(iv) For every closed subgroup $U$ of $G, U$ and $G / U$ are $\sigma$-compact.

(v) There is a closed subgroup $U$ of $G$ such that $U$ and $G / U$ are $\sigma$-compact.

(vi) $G / G_{0}$ is $\sigma$-compact.

Proof. (i) and (ii) are equivalent for every locally compact space. The following are obvious: (ii) $\rightarrow$ (iii) $\rightarrow$ (i) $\rightarrow$ (iv) $\rightarrow$ (v) and (iv) $\rightarrow$ (vi). Assume (v), and let $\left(K_{n}\right)_{n \in N}$ be a compact countable cover of $G / U$. Let $\left(M_{\alpha}\right)_{\alpha \in A}$ be an open, relatively compact cover of $G$. Then finitely many of the open sets $M_{\alpha} U$ cover $K_{n}$, i.e., $K_{n}$ is of the form $K_{n}^{\prime} U$ with $K_{n}^{\prime}$ compact in $G$. If $\left(K_{m}^{\prime \prime}\right)_{m \in N}$ is a countable compact cover of $U$, then $\left(K_{n}^{\prime} K_{m}^{\prime \prime}\right)_{n, m \in N}$ is a countable compact cover of $G$. Hence $(\mathrm{v}) \rightarrow(\mathrm{i})$. As the identity component $G_{0}$ of $G$ is compactly generated, it is $\sigma$-compact, whence (vi) $\rightarrow(v)$.

Lemma 2.3. Let $E(G)$ denote the set of all continuous positive definite functions on $G$ with supremum equal to 1 . Then the topology of $G$ is the coarsest of all topologies with respect to which the elements of $E(G)$ are continuous.

A proof can be found in [5]. Its idea is to check that for any symmetric neighborhood $V$ of the identity, a suitable multiple of the convolution square of the characteristic function of $V$ is an element of $E(G)$ with support in $V^{2}$. 
Notation 2.4. Henceforth, $G$ is a group, and $\rho$ and $\tau$ are two topologies on $G$ with respect to each of which $G$ is a locally compact group. We denote the locally groups arising this way by $G_{o}$ and $G_{\tau}$, respectively. Their identity components, considered as abstract groups, are $G_{\rho, 0}$ and $G_{\tau, 0}$, respectively. If $U$ is a subgroup of $G, U_{\rho}$ denotes the topological group obtained by inducing $\rho$ on $U$. If $U$ is a (normal) subgroup of $G, G_{\rho} / U$ denotes the topological space (topological group) which is the quotient of the topological group $G_{\rho}$ by $U$. We write $G_{\rho} \simeq G_{\tau}\left(G_{\rho} / U \simeq G_{\tau} / U\right)$, if $G_{\rho}$ and $G_{\tau}\left(G_{\rho} / U\right.$ and $\left.G_{\tau} / U\right)$ are equal as topological groups (topological spaces).

Lemma 2.5. Assume $G_{\rho}$ and $G_{\tau}$ are both $\sigma$-compact groups. Then, if there exists a Hausdorff topology on $G$ coarser than both $\rho$ and $\tau, G_{\rho} \simeq G_{\tau}$.

A proof based on the Baire category theorem can be found in $[16$, p. 58].

Lemma 2.6. Again let $G_{\rho}$ and $G_{\tau}$ be locally compact groups with the same underlying group $G$ such that there exists a Hausdorff topology coarser than both $\rho$ and $\tau$. Let $U$ be a subgroup of $G$, closed in $G_{\rho}$ and $G_{\tau}$ such that $U_{\rho} \simeq U_{\tau}$ and $G_{\rho} / U \simeq G_{\tau} / U$. If $U$ is $\sigma$-compact, then $G_{\rho} \simeq G_{\tau}$.

Proof. Let $V$ be an open symmetric neighborhood of $e$ in $G_{o}$. Then there is a symmetric neighborhood $V^{\prime}$ of $e$ in $G_{\tau}$ such that $V U=$ $V^{\prime} U$ and $V \cap U=V^{\prime} \cap U . \quad U$ can be covered by countably many left translates $u_{n} V \cap U, u_{n} \in U, n \in N$ (2.2). Let $V^{\prime}{ }_{n}=V^{\prime} \cup\left\{u_{1}, u_{1}^{-1}\right\} \cup\left\{u_{2}\right.$, $\left.u_{2}^{-1}\right\} \cup \cdots \cup\left\{u_{n}, u_{n}^{-1}\right\}$. Then $\widetilde{V}_{n}^{\prime}:=\bigcup_{m}\left(V_{n}^{\prime}\right)^{m}$ is an open $\sigma$-compact subgroup of $G_{\tau}$, hence so is $\widetilde{V}^{\prime}:=\bigcup_{n} \widetilde{V}_{n}^{\prime}$. Let us show that $V \subset \widetilde{V}^{\prime}$. If $v \in V$, there exists $v^{\prime} \in V^{\prime}$ and $u \in U$ such that $v=v^{\prime} u$. Let $u \in u_{n} V \cap U$. Then $v \in\left(V_{n}^{\prime}\right)^{3} \subset \tilde{V}^{\prime}$. It is obvious that the group $\tilde{V}$ constructed analogously from $V$ is contained in $\tilde{V}^{\prime}$, and even that the two groups $\widetilde{V}^{\prime}$ and $\widetilde{V}$ coincide. As they are both $\sigma$-compact in their respective topologies, they are equal as topological groups (2.5). Hence $V$ and $V^{\prime}$ are open both in $G_{\rho}$ and $G_{\tau}$.

3. Weak equivalence and first properties. Throughout this section we resume the notation 2.4 and add the following: If $A$ is a $C^{*}$-algebra, $C(A)$ is the convex cone of positive linear functionals on $A$ with norm not exceeding $1 . E(A)$ is the subset of $C(A)$ of states, i.e., of functionals of norm $1 . P(A)$ is the set of pure states. If $T$ is an irreducible representation of $A, P_{T}(A)$ denotes the set of (pure) 
states whose canonically associated representation is unitarily equivalent to $T$.

$C(A), E(A), P_{T}(A)$ are furnished with the weak*-topology inherited from $A^{\prime}$. The topological spaces so obtained are again denoted $C(A)$, $E(A)$ etc. $C(A)$ is a compact space.

If $A$ is the $C^{*}$-algebra $A(G)$ of a locally compact group $G$, we sometimes write $C(G), \cdots, P_{T}(G)$ instead and identify these spaces in the usual manner with spaces of continuous positive definite functions on $G$ (cf. $[4, \S 13])$. If we want to distinguish the topology $\rho$ of $G$, we also write $C\left(G_{\rho}\right)$ etc. By a representation of a group, we always mean a unitary representation $T$ in some Hilbert space $\mathscr{H}_{T}$.

DEFINITION 3.1. The locally compact groups $G_{\rho}, G_{\tau}$ (see 2.4 above) are called weakly equivalent, denoted $G_{\rho} \sim G_{\tau}$, if every irreducible representation of $G$ continuous with respect to $\rho$ is also continuous with respect to $\tau$ and vice versa.

In view of the canonical correspondence between continuous irreducible representations of a locally compact group $G$ and continuous irreducible positive definite functions on $G$, we can restate this definition thus: $G_{\rho} \sim G_{\tau}$ if and only if $P\left(G_{\rho}\right)=P\left(G_{\tau}\right)$. It is obvious that $\sim$ is an equivalence relation.

\section{Lemma 3.2. Let $G_{\tau} \sim G_{\rho}$.}

(i) If $U$ is subgroup of $G$, open in $G_{\tau}$, then $U$ is closed in $G_{\rho}$, and every continuous irreducible representation of $U_{\tau}$ is continuous with respect to $\rho$.

(ii) If $N$ is a normal subgroup of $G$ closed in $G_{\rho}$, then $N$ is also closed in $G_{\tau}$, and $G_{\rho} / N \sim G_{\tau} / N$.

(iii) There is a Hausdorff topology on $G$ coarser than both $\rho$ and $\tau$.

Proof. The $C^{*}$-algebra $A\left(U_{\tau}\right)$ of $U_{\tau}$ is a subalgebra of $A\left(G_{\tau}\right)$, and its irreducible representations are the restrictions of irreducible representations of $A\left(G_{\tau}\right)([4, \S 2.10])$. That is, the continuous irreducible representations of $U_{\tau}$ are the restrictions of irreducible continuous representations of $G_{\tau}$. They are, therefore, continuous on $U_{\rho}$.

This argument also shows that the continuous irreducible positive definite functions of $U_{\tau}$ are the restrictions to $U$ of those of $G_{\tau}$. Consider the convex set $E_{U}\left(G_{\tau}\right)$ of continuous positive definite functions on $G_{\tau}$ which equal 1 on $U . E_{U}\left(G_{\tau}\right)$ is obviously compact in the weak*topology of $A\left(G_{\tau}\right)^{\prime}$. It contains the characteristic function $\chi_{U}$ of $U$. By the theorem of Krein-Milman, it is the closed convex hull of its extreme points $P_{U}\left(G_{\tau}\right)$. Let $f=f_{1}+f_{2}$ be an element of $P_{U}\left(G_{\tau}\right)$, where 
the $f_{i}$ are continuous positive definite on $G_{\tau}$. Then the restrictions to $U$ of the $f_{i}$ are proportional, and as $f$ is extreme in $E_{U}\left(G_{\tau}\right)$, the $f_{i}$ are proportional. Hence $f$ is an irreducible positive definite function on $G_{\tau}$, and it follows that the elements of $P_{U}\left(G_{\tau}\right)$ are $\rho$-continuous. As $\chi_{U} \in E_{U}\left(G_{\tau}\right), U$ is the intersection of the sets $\left\{s \in G \mid f(s)=1, f \in P_{U}\left(G_{\tau}\right)\right\}$, which are closed in $G_{\rho}$. Hence $U$ is closed in $G_{\rho}$, whence (i). Statement (ii) says that the continuous irreducible representations of $G_{\rho}$ or $G_{\tau}$ which contain $N$ in their kernel are coincident, and this is obvious. The coarsest topology $\sigma$ such that all $\rho$-continuous irreducible representations of $G$ are $\sigma$-continuous meets the requirements of (iii).

Corollary. Let $\rho, \tau$ be topologies on $G$ which make it into a locally compact group; and let $U$ be a subgroup of $G$. If $U$ is open both in $G_{\tau}$ and $G_{\rho}$, then
(i) $G_{\tau} \simeq G_{\rho}$ if and only if $U_{\tau} \simeq U_{\rho}$ and
(ii) $G_{\tau} \sim G_{\rho}$ if and only if $U_{\tau} \sim U_{\rho}$.

4. Weak equivalence with a compact group. We start with some lemmas on compact convex sets. In the first two cases we assume we are given a convex compact subset $C$ of some locally convex vector space together with a complex vector space $A$ of affine-linear continuous functions on $C$ which separates the points of $C$.

With these data, we define a metric $d_{A}$ on $C$ by putting $d_{A}(x, y)=$ $\sup \{|a(x)-a(y)| ; a \in A,|a| \leqq 1\},|a|$ being the sup-norm of $a \in A$.

Definition. Let $K$ be a subset of $C$ and $\hat{K}$ its closed convex hull. We say that $K$ is $A$-uniform, if the set of finite convex-linear combinations of extreme points of $\hat{K}$ is dense in $\hat{K}$ with respect to the topology defined by $d_{A}$.

Lemma 4.1. Let $P$ be the set of extreme points of $C$. If $P$ is the union of countably many disjoint, A-uniform sets $K_{n}, n \in N$, then $C$ is A-uniform.

Proof. Let $x \in C$. According to Choquet's theorem ([1, p. 57] or [3, Lemma 28]), there is a probability measure $\mu$ on $C$ with barycenter $x$ and concentrated on $P$. Let $\chi_{n}, n=1,2, \cdots$, be the characteristic function of $K_{n}$ and put $\mu_{n}=\mu \chi_{n}$. Then $\sum\left|\mu_{n}\right|=1,\left|\mu_{n}\right|^{-1} \mu_{n}$ is a probability measure on $C$, which has a barycenter $x_{n} \in \hat{K}_{n}$ [1]. (We may assume that all the $\mu_{n}$ are $\neq 0$, omitting the others.)

Let an $\varepsilon>0$ be given, $\varepsilon<1 / 2$, say.

As $K_{n}$ is compact and contained in $P$, it coincides with the set of extreme points of $\hat{K}_{n}$, hence there is a convex-linear combination $y_{n}$ of points of $K_{n}$ such that $d_{A}\left(x_{n}, y_{n}\right)<\varepsilon$. 
Let $n^{\prime}$ be such that $1 \geqq m:=\sum_{n=1}^{n \prime}\left|\mu_{n}\right|>1-\varepsilon$ and put $y=$ $m^{-1} \sum_{n=1}^{n \prime}\left|\mu_{n}\right| y_{n}$. Then $y$ is a finite convex-linear combination of points of $P$, and for all $a \in A$ with $|a| \leqq 1$ we have

$$
\begin{aligned}
|a(x)-a(y)| & =\left|\mu(a)-m^{-1} \sum_{n=1}^{n \prime}\right| \mu_{n}\left|a\left(y_{n}\right)\right| \\
& \leqq\left|\sum_{n=1}^{n \prime} \mu_{n}(a)-m^{-1} \sum_{n=1}^{n \prime}\right| \mu_{n}\left|a\left(y_{n}\right)\right|+\left|\sum_{n=n^{\prime}+1}^{\infty} \mu_{n}(a)\right| \\
& \leqq \sum_{n=1}^{n \prime}\left|\mu_{n}\right|\left|a\left(x_{n}\right)-m^{-1} a\left(y_{n}\right)\right|+\varepsilon \leqq \varepsilon+m^{-1} \varepsilon+\varepsilon \leqq 4 \varepsilon .
\end{aligned}
$$

That is, $d_{A}(x, y) \leqq 4 \varepsilon$, and the lemma is proven.

4.2. We now return to the notation of 3 . Let $A$ be a $C^{*}$-algebra and view it in the obvious manner as a vector space of continuous affine-linear functions on $C(A)$. Let $T$ be an irreducible representation of $A$ in some Hilbert space $\mathscr{H}_{T}$ such that $T(a)$ is a compact operator for all $a \in A$. Then $T(A)=\mathscr{L} \mathscr{C}\left(\mathscr{C}_{T}\right)$, the algebra of all compact operators of $\mathscr{K}_{T}([4, \S 4])$.

Lemma. (i) The closed convex subcone $C_{T}(A)$ of $C(A)$ spanned by 0 and $P_{T}(A)$ is A-uniform.

(ii) If $T$ is finite-dimensional, then $P_{T}(A)$ is compact, and every element of $C_{T}(A)$ is a finite linear combination of pure states in $P_{T}(A)$.

Proof. (cf. [4, §4]). Let $x \in C_{T}(A)$. As a pointwise limit of linear functionals vanishing on $\operatorname{ker} T, x$ also vanishes on $\operatorname{ker} T$ and can be viewed as a positive linear functional on $A / \operatorname{ker} T \simeq \mathscr{L} \mathscr{C}\left(\mathscr{C}_{T}\right)$. Hence it is of the form $x: a \rightarrow \sum \lambda_{n}\left(T(a) \xi_{i} \mid \xi_{i}\right)$, where $\lambda_{i}>0, \sum_{i=1}^{\infty} \lambda_{i} \leqq 1$ and $\left\{\xi_{i}\right\}$ is an orthonormal set of vectors in $\mathscr{H}_{T}$. Hence $x$ lies in the convex compact cone spanned by 0 and the at-most-countably-many elements $a \rightarrow\left(T(a) \xi_{i} \mid \xi_{i}\right)$ of $P_{T}(A)$. This cone is $A$-uniform according to the preceding lemma, i.e., $x$ is in the convex and $d_{A}$-closed hull of $P_{T}(A) \cap\{0\}$. (ii) is now obvious.

4.3. Now let $A$ be the $C^{*}$-algebra of the locally compact group $G$. $C(A)$ is identified as usual with the set of continuous positive definite functions on $G$ with $|x|_{\infty}=x(e) \leqq 1$.

Lemma. $\quad d_{A}(x, y) \geqq|x-y|_{\infty}$ for $x, y \in C(A) \subset L^{\infty}(G)$. That is, the topology defined by $d_{A}$ on $C(A)$ is finer than the topology of uniform convergence on $G$.

Proof. $\quad d_{A}(x, y)=\sup \{(x-y \mid a) ; a \in A,|a| \leqq 1\}$ 


$$
\begin{aligned}
& =\sup \left\{(x-y \mid \Phi) ; \Phi \in L^{1}(G),|\Phi| \leqq 1\right\} \\
& \geqq \sup \left\{(x-y \mid \Phi) ; \Phi \in L^{1}(G),|\Phi|_{1} \leqq 1\right\} \\
& =\sup \{|x(s)-y(s)| ; s \in G\}=|x-y|_{\infty},
\end{aligned}
$$

since $L^{1}(G)$ is dense in $A$ and since the norm $|\Phi|$ of an element $\Phi$ of $L^{1}(G)$ in $A$ does not exceed its norm $|\Phi|_{1}$ in $L^{1}(G)$.

Lemma 4.4. Let $G_{\rho} \sim G_{\tau}$ and suppose $G_{\rho}$ compact. Let $U$ be a subgroup of $G$ which is a closed $G_{\delta}$ in $G_{\rho}$. Then a positive definite function, constant on $U$, is $\rho$-continuous if and only if it is $\tau$ continuous.

Proof. Let ${ }_{V} \hat{G}_{\rho}$ denote the set of all equivalence classes $\hat{T}$ of continuous irreducible representations of $G_{\rho}$ such that at least one of the positive definite functions associated to $\hat{T}$ is constant on $U$, and for every such $\hat{T}$ select a representative $T$ contained in the left regular representation of $G_{\rho}([11, \mathrm{p} .164])$. Furthermore, for every $T$ so obtained select a (continuous) function $\Phi_{T} \in \mathscr{C}_{T} \subset L^{2}\left(G_{\rho}\right)$ of 2-norm 1, such that the corresponding positive definite function $x_{T}: s \rightarrow\left(T(s) \phi_{T} \mid \phi_{T}\right)$ is constant on $U$; i.e., such that $\phi_{T}$ is constant on right cosets $U t, t \in G$. As all the $\mathscr{H}_{T}$ 's are finite-dimensional, it is clear that the dimension of their Hilbert space span in $L^{2}\left(G_{\rho}\right)$ is equal to the dimension of the span of the $\phi_{T}$ in $L^{2}\left(G_{\rho}\right)$, which again is the cardinality of ${ }_{U} \widehat{G}_{\rho}{ }^{2)}$. We want to show that this cardinality is countable. Let $V_{n}, n \in N$, be a sequence of compact neighborhoods of the identity such that $U=\cap U V_{n}$. Such a sequence exists since $U$ is a compact $G_{i}$. For every $n \in N$, there is a finite number $m_{n}$ of elements $s_{n}^{m}$ of $G$ such that the right translates $U V_{n} s_{n}^{m}, m=1, \cdots m_{n}$, cover $G$. Let $\chi_{n}^{m}$ denote the countably many characteristic functions of the sets $U V_{n} s_{n}^{m}$. If the inner products $\left(\Phi_{T} \mid \chi_{n}^{m}\right)$ all vanish for the function $\Phi_{T}$, then $\Phi_{T}=0$. Indeed, if $\Phi_{T}(s)=0$ say $\Phi_{T}(s)>0$, then $\Phi_{T}>0$ in one of the sets $U V_{n} s_{n}^{m}$, and $\left(\Phi_{T} \mid \chi_{n}^{m}\right)>0$. That is, we have shown that ${ }_{U} \widehat{G}_{\rho}$ is countable, or else, that there are at most countably many $\widehat{T}$ 's in $\hat{G}_{\rho}$ with one of the associated positive definite functions constant on $U$. As $G_{\rho} \sim G_{\tau}$, the same holds for $G_{\tau}$.

Now let $C_{U}^{\prime}\left(G_{\rho}\right)$ denote the set of all $\rho$-continuous positive definite functions on $G$, constant on $U$ and of norm not exceeding $1 . C_{U}^{\prime}\left(G_{\rho}\right)$ is a convex cone, compact in the weak*-topology of $A\left(G_{\rho}\right)^{\prime}$. It is clearly contained in the compact convex cone $C_{U}\left(G_{\rho}\right)$ spanned by 0 and the sets $P_{T}\left(G_{\rho}\right), \widehat{T} \in{ }_{U} \widehat{G}_{\rho}={ }_{U} \widehat{G}_{\tau}$. Lemma 4.2 tells that these sets are $A\left(G_{\rho}\right)$ uniform; according to Lemma 4.1 and the preceding argument, $C_{U}\left(G_{\rho}\right)$

2) This is strictly true only if ${ }_{U} \hat{G}_{\rho}$ is infinite. If it is finite, there is nothing to prove. 
is $A\left(G_{\rho}\right)$-uniform. With Lemma 4.3 we can state: every $\rho$-continuous positive definite function $x$ on $G$, constant on $U$, is a uniform limit on $G$ of convex-linear combinations of irreducible $\rho$-continuous positive definite functions on $G$. But the latter are also $\tau$-continuous, hence so is $x$. Interchanging $\rho$ and $\tau$ in this argument, we see that, indeed, if $x$ is $\tau$-continuous and constant on $U$, it is also $\rho$-continuous.

CoROLlary. If $G_{\tau} \sim G_{\rho}$ and $G_{\rho}$ is compact and metrizable, then $G_{\rho} \simeq G_{\tau}$. In fact, $\rho$ is the coarsest of all topologies on $G$ with respect to which the elements of $P\left(G_{\rho}\right)$ are continuous.

For then $\{e\}$ is a $G_{\hat{o}}$, and Lemma 2.3 yields the result.

Proposition 4.5. If $G_{\tau} \sim G_{\rho}, G_{\rho}$ is compact, and $U$ is an open subgroup of $G_{\tau}$, then there exists a smallest open subgroup $\breve{U}$ of $G_{\rho}$ containing $U$.

Proof. Let $\mathscr{U}$ be the set of all open subgroups of $G_{\rho}$ containing $U$, ordered by inclusion. Let $\left(U_{\alpha}\right)_{\alpha \in A}$ be a chain of distinct elements in $\mathscr{C}$. Then $A$ is at most countable. Indeed, the mapping $\alpha \rightarrow$ (index of $U_{\alpha}$ in $G$ ) is one-to-one from $A$ into $N$. Let $U^{\prime}=\cap U_{\alpha}$. Then $U^{\prime}$ is a compact $G_{\delta}$, open in $G_{\tau}$, as it contains $U$. The characteristic function of $U^{\prime}$ is constant on $U$, positive definite, and $\tau$-continuous. Hence it is $\rho$-continuous (4.4), and $U^{\prime}$ is $\rho$-open. We have shown that $\mathscr{C}$ is inductively ordered and hence has a smallest element, $\breve{U}$.

5. Weakly equivalent topologies on G, one of which is the topology of a Lie group.

Proposition. If $G_{\rho}$ is a Lie group and $G_{\tau} \sim G_{\rho}$, then $G_{\tau} \simeq G_{\rho}$.

Proof. As $G_{\rho} / G_{\rho, 0}$ is discrete and weakly equivalent to $G_{:} / G_{\rho, 0}$, Proposition 1 yields that $G_{\rho, 0}$ is open in $G_{\tau}$ also. Once we know that $G_{\rho, 0, \rho} \simeq G_{\rho, 0, \tau}$, the proposition is established (cf. 2.5). That is, we may assume without restriction that $G_{\rho}$ is connected (cf. Corollary 3.2).

$G_{\tau, 0}$ is a closed subgroup of $G_{\rho}$, hence $\sigma$-compact with respect to both $\rho$ and $\tau$ (Lemma $2.2 \mathrm{iv}$ )). In view of Lemmas 3.2, 2.5, and 2.6, we need only show that $G_{\tau} / G_{\tau, 0} \simeq G_{\rho} / G_{\tau, 0}$. That is, we may assume additionally that $G_{\tau}$ is totally disconnected.

Let $U$ be a compact open subgroup of $G_{\tau}$. According to Lemma 3.2 (i), $U$ is a closed subgroup of $G_{\rho}$, hence $U_{\rho}$ is a Lie subgroup of $G_{\rho}$, and, therefore, according to Lemma 2.5, discrete in both $\rho$ and $\tau$. Hence $G_{\tau}$ is discrete, and Proposition 1 yields $G_{\rho} \simeq G_{\tau}$. 
6. Weakly equivalent totally disconnected topologies on G.

Lemma 6.1. Let $G_{\tau} \sim G_{o}, \tau$ finer than $\rho$, and $\rho$ totally disconnected. Then $G_{\tau} \simeq G_{\rho}$.

Proof. There is a compact open subgroup of $G_{\rho}$, open also in $G_{\tau}$ as $\tau \supseteqq \rho$, so Corollary 3.2 tells that it is no restriction to assume that $G_{\rho}$ is compact.

$G_{\tau}$ is totally disconnected, too, and hence has a basis at $e$ of open compact subgroups. Let $U$ be one of them. There exists a smallest open subgroup $\check{U}$ of $G_{\rho}$ containing $U$ (Proposition 4.5). Now $G_{\rho}$ has a basis at $e$ of open normal subgroups, $\mathscr{N}$. We have $\breve{U} \subset U N$ for all $N \in \mathscr{N}$, hence $\breve{U} \subset \cap N U=U: U$ is also open in $G_{\rho}$, and $G_{\tau} \simeq G_{\rho}$.

LEMMA 6.2. Let $G_{\tau} \sim G_{\rho}, \tau \supseteqq \rho$, and $G_{\tau}$ totally disconnected. Then $G_{\rho} \simeq G_{\tau}$.

Proof. There is an open subgroup of $G_{\rho}$, open also in $G_{\tau}$, which is almost connected. According to Corollary 3.2 it is no restriction to assume that $G_{\rho}$ is almost connected. We want to show that $G_{\rho}$ is also totally disconnected, thus reducing the lemma to the preceding one. Now for every neighborhood $V$ of $e$ in $G_{\rho}$ there is a compact normal subgroup $N$ in $V$ such that $G_{\rho} / N$ is a Lie group $([14$, p. 175]). We have $G_{\tau} / N \sim G_{\rho} / N$ (Lemma 3.2), hence $G_{\tau} / N \simeq G_{\rho} / N$ (Proposition 54). But $G_{\tau} / N$ is totally disconnected (cf. 2.1), hence discrete, i.e., $N$ is open in $G_{\rho}$. As $G_{\rho, 0}$ is the intersection of all open subgroups of $G_{\rho}, G_{\rho, 0}=\{e\}$.

Proposition 6.3. Let $G_{\rho} \sim G_{:}$, with $G_{\rho}$ totally disconnected and $\sigma$-compact. Then $G_{\tau} \simeq G_{\rho}$.

Proof. Let $U$ be an open compact subgroup of $G_{\rho}$. Then $U$ is closed in $G_{\tau}$, (Lemma 3.2), and a countable number of left translates of $U$ covers $G_{\tau}$ (Lemma 2.2 (iii)). According to the Baire category theorem, one of them and therefore all of them are open in $G_{\tau}$. Letting $U$ run through a basis at $e$ of $G_{\rho}$, we see that $\tau$ is finer than $\rho$. The preceding lemma yields the result.

\section{Comparable topologies on G.}

Proposition. Let $G_{\tau} \sim G_{\rho}$ and $\tau \supseteqq \rho$. Then $G_{\tau} \simeq G_{\rho}$.

Proof. $G_{\tau, 0}$ is contained in $G_{\rho, 0}$ and hence (Lemma 2.2 (iv)) it is $\sigma$-compact with respect to both topologies. Thus $G_{\tau, 0, \tau} \simeq G_{\tau, 0, \rho}$, and 
in view of Lemma 2.6, we may assume $G_{\tau}$ to be totally disconnected. That is, the proposition is reduced to Proposition 6.2.

\section{8. $\sigma$-compact topologies.}

Proposition. Let $G_{\rho}$ be $\sigma$-compact and $G_{\rho} \sim G_{\tau}$. Then $G_{\rho} \simeq G_{\tau}$.

Proof. We have $G_{\tau} / G_{\rho, 0} \simeq G_{\rho} / G_{\rho, 0}$ according to 6.3. This shows that an open almost connected subgroup of $G_{\rho}$ is also open in $G_{\tau}$, and we may assume $G_{\rho}$ to be almost connected (cf. 3.2). Let $V$ be a neighborhood of $e$ in $G_{\rho}$ and $N$ a compact normal subgroup such that $G_{\rho} / N$ is a Lie group. Then $G_{\tau} / N \simeq G_{\rho} / N$ (cf. 5.5) and, in particular $G_{\tau} / N G_{\tau, 0} \simeq G_{\rho} / N G_{\tau, 0}$. Section 2.1 shows that $G_{\tau} / N G_{\tau, 0}$ is discrete, hence $G_{\rho, 0} \subset N G_{\tau, 0}$. Since $V$ can be chosen arbitrarily small, $G_{\rho, 0} \subset G_{\tau, 0}$. As in the proof of Proposition 7.1, we divide by $G_{\rho, 0}$ and thus reduce the proof to Proposition 6.3.

\section{Summary.}

THEOREM. Let $G$ be a locally compact group with respect to two topologies. If the irreducible unitary representations continuous with respect to either topology coincide, then so do the topologies in the following cases:

(i) The topogies are comparable;

(ii) There exists a normal subgroup $N$ of $G$, open and $\sigma$-compact in one of the topologies.

Indeed, (i) is Proposition 7, while (ii) follows from Proposition 8 and Corollary 3.2, since $N$ is, in fact, open in both topologies (Proposition 1).

The class of groups covered by (ii) is slightly larger than the class of "manageable" groups treated by Greenleaf [7], Leptin [11], [12], and others in their investigations of properties equivalent to amenability.

\section{REFERENCES}

1. H. Bauer, Konvexitaet in topologischen Vektorraeumen, lecture notes, Hamburg, $1963 / 64$.

2. K. Bichteler, On the existence of noncontinuous irreducible representations of a locally compact group, Jnveutiones Math. 6 (1968), 159-162.

3. G. Choquet \& P. A. Meyer, Existence et unicite des representations integrales dans les convexes compacts quelconques, Ann. Inst. Fourier, Grenoble 13 (1963), 139-154.

4. J. Dixmier, Les $C^{*}$-Algebres et leurs representations, Gauthier-Villars, Paris, 1964.

5. J. Ernest, A new group algebra for locally compact groups, Amer. J. Math. 86 (1964), 467-491. 
6. I. Glicksberg, Uniform houndedness for groups, Canad. J. Math. 14 (1962).

7. F. P. Greenleaf, Folner conditions for locally compact groups, preprint, Berkeley, Calif., 1967.

8. S. Helgason, Differential geometry and symmetric spaces, Academic Press, 1962.

9. E. Hewitt, A remark on characters of locally compact Abelian groups, Fund. Math. 53 (1964), 55-64.

10. E. Hewitt \& K. Ross, Abstract harmonic analysis, I, Springer, Berlin-GoettingenHeidelberg, 1963.

11. H. Leptin, On locally compact groups with an invariant mean (to appear).

12. - On a certain invariant of a locally compact group, Bull. Amer. Math. Soc. 72 (1966), 870-874.

13. L. Loomis, An introduction to abstract harmonic analysis, van Nostrand, Princeton, 1953.

14. D. Montgomery. \& L. Zippin, Topological transformation groups, Interscience Publishers (Wiley), New York, 1966.

15. K. A. Ross, Closed subgroups of locally compact Abelian groups, Fund. Math. 56 (1965), 241-244.

Received June 25, 1968, and in revised form May 21, 1969. This Research was sponsored by the Air Force Office of Scientific Research under Grant AF-AFOSR-903-67 and by the National Aeronautics and Space Administration under Grant NGR 44-004042 and Grant NSG-269-62.

UNIVERSITY OF TEXAS

Austin, Texas 



\title{
PACIFIC JOURNAL OF MATHEMATICS
}

\author{
EDITORS
}

\author{
H. ROYDEN \\ Stanford University \\ Stanford, California \\ RICHARD PIERCE \\ University of Washington \\ Seattle, Washington 98105
}

\author{
J. DUGUNDJI \\ Department of Mathematics \\ University of Southern California \\ Los Angeles, California 90007 \\ BASIL GORDON \\ University of California \\ Los Angeles, California 90024
}

\section{ASSOCIATE EDITORS}

E. F. BECKENBACH
B. H. NeumanN

F. WOLF
K. YoshidA

\section{SUPPORTING INSTITUTIONS}

\author{
UNIVERSITY OF BRITISH COLUMBIA \\ CALIFORNIA INSTITUTE OF TECHNOLOGY \\ UNIVERSITY OF CALIFORNIA \\ MONTANA STATE UNIVERSITY \\ UNIVERSITY OF NEVADA \\ NEW MEXICO STATE UNIVERSITY \\ OREGON STATE UNIVERSITY \\ UNIVERSITY OF OREGON \\ OSAKA UNIVERSITY \\ UNIVERSITY OF SOUTHERN CALIFORNIA
}

\author{
STANFORD UNIVERSITY \\ UNIVERSITY OF TOKYO \\ UNIVERSITY OF UTAH \\ WASHINGTON STATE UNIVERSITY \\ UNIVERSITY OF WASHINGTON

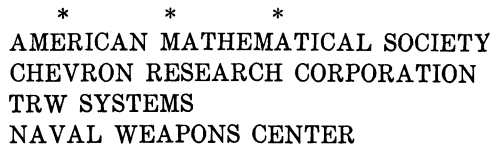

The Supporting Institutions listed above contribute to the cost of publication of this Journal, but they are not owners or publishers and have no responsibility for its content or policies.

Mathematical papers intended for publication in the Pacific Journal of Mathematics should be in typed form or offset-reproduced, double spaced with large margins. Underline Greek letters in red, German in green, and script in blue. The first paragraph or two must be capable of being used separately as a synopsis of the entire paper. It should not contain references to the bibliography. Manuscripts, in duplicate if possible, may be sent to any one of the four editors. Please classify according to the scheme of Math. Rev. 36, 1539-1546. All other communications to the editors should be addressed to the managing editor, Richard Arens, University of California, Los Angeles, California, 90024.

50 reprints are provided free for each article; additional copies may be obtained at cost in multiples of 50 .

The Pacific Journal of Mathematics is published monthly. Effective with Volume 16 the price per volume (3 numbers) is $\$ 8.00$; single issues, $\$ 3.00$. Special price for current issues to individual faculty members of supporting institutions and to individual members of the American Mathematical Society: $\$ 4.00$ per volume; single issues $\$ 1.50$. Back numbers are available.

Subscriptions, orders for back numbers, and changes of address should be sent to Pacific Journal of Mathematics, 103 Highland Boulevard, Berkeley, California, 94708.

PUBLISHED BY PACIFIC JOURNAL OF MATHEMATICS, A NON-PROFIT CORPORATION

Printed at Kokusai Bunken Insatsusha (International Academic Printing Co., Ltd.), 7-17, Fujimi 2-chome, Chiyoda-ku, Tokyo, Japan. 


\section{Pacific Journal of Mathematics}

\section{Vol. 31, No. $3 \quad$ BadMonth, 1969}

George E. Andrews, On a calculus of partition functions .................. 555

Silvio Aurora, A representation theorem for certain connected rings ............ 563

Lawrence Wasson Baggett, A note on groups with finite dual spaces ............. 569

Steven Barry Bank, On majorants for solutions of algebraic differential equations in regions of the complex plane ............................... 573

Klaus R. Bichteler, Locally compact topologies on a group and the corresponding continuous irreducible representations ......................... 583

Mario Borelli, Affine complements of divisors ....................... 595

Carlos Jorge Do Rego Borges, A study of absolute extensor spaces ............. 609

Bruce Langworthy Chalmers, Subspace kernels and minimum problems in Hilbert

spaces with kernel function ...................................... 619

John Dauns, Representation of L-groups and F-rings................. 629

Spencer Ernest Dickson and Kent Ralph Fuller, Algebras for which every

indecomposable right module is invariant in its injective envelope ...........

Robert Fraser and Sam Bernard Nadler, Jr., Sequences of contractive maps and fixed

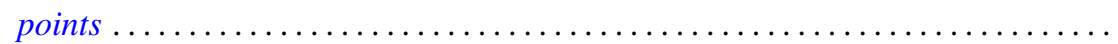

Judith Lee Gersting, A rate of growth criterion for universality of regressive

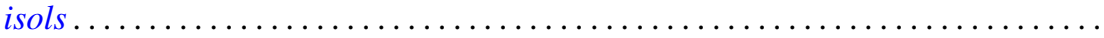

Robert Fred Gordon, Rings in which minimal left ideals are projective ............

Fred Gross, Entire functions of several variables with algebraic derivatives at certain algebraic points

W. Charles (Wilbur) Holland Jr. and Stephen H. McCleary, Wreath products of ordered permutation groups .........................

W. J. Kim, The Schwarzian derivative and multivalence .................. 717

Robert Hamor La Grange, Jr., On $(\mathrm{m}-\mathrm{n})$ products of Boolean algebras ......... 725

Charles D. Masiello, The average of a gauge ........................ 733

Stephen H. McCleary, The closed prime subgroups of certain ordered permutation

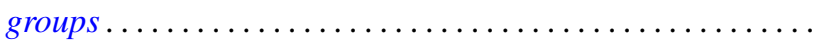

Richard Roy Miller, Gleason parts and Choquet boundary points in convolution measure algebras ...............................

Harold L. Peterson, Jr., On dyadic subspaces ........................ 773

Derek J. S. Robinson, Groups which are minimal with respect to normality being intransitive........................................... 777

Ralph Edwin Showalter, Partial differential equations of Sobolev-Galpern type . . . 787

David Slepian, The content of some extreme simplexes ................... 795

Joseph L. Taylor, Noncommutative convolution measure algebras ............. 809

B. S. Yadav, Contractions of functions and their Fourier series ............... 827

Lindsay Nathan Childs and Frank Rimi DeMeyer, Correction to automorphisms of separable algebras" ....................... 833

Moses Glasner and Richard Emanuel Katz, Correction to: "Function-theoretic degeneracy criteria for Riemannian manifolds".............

Satish Shirali, Correction to: "On the Jordan structure of complex Banach

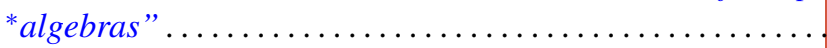

